\section{Contain and capture dental} aerosols at their source

Move beyond just PPE protections with the only system specifically designed for the capture of dental aerosols and splatter in dental working conditions: the Rhondair Aerosol Capture System.

Safely reduce your fallow period and risks by capturing all oral and nasal exhalations, without losing direct intraoral vision or access.

An exceptionally powerful fan draws away suspended aerosols to a medicalgrade $\mathrm{H} 13 \mathrm{HEPA}$ filter at $4,000 \mathrm{~L} / \mathrm{min}$ for unmatched performance. The filter captures $99.95 \%$ or more of all particles - including the size ranges that viruses, COVID-19, and dental aerosols exist within.

At just $52 \mathrm{~dB}$ at the funnel, the Rhondair is similar in volume to a heat pump and significantly quieter than standard HVE systems.

Single-use shields and sleeves provide the best infection control available today. Faster and safer than disinfecting other systems, with less chance of crosscontamination. Recyclable shields and sleeves ensure safety doesn't come at a cost to the environment.

The prevention of just one lost day of production, whether it be from COVID19 or common cold/flu, will pay for the Rhondair system.

Units are available now with free shipping.

Contact 0800680 0636, email info@ rhondium.com, or visit rhondium.co.uk to learn more.

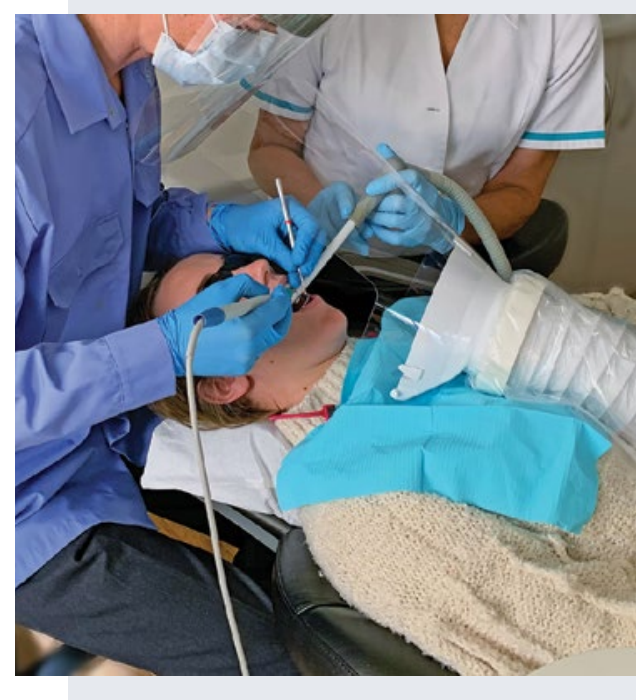

\title{
Plaque prevention at home or in practice
}

Kerr is excited to introduce PlakACT line with Chlorhexidine, inspired by proven formulations available in the Swiss market for 40 years: your new ally in oral health.

Excessive bacterial deposits generate an unhealthy oral environment that adversely affects the mucous membranes. This should not be ignored. It's important to act fast and follow an accurate oral hygiene regime to prevent an escalation of the symptoms.

The new PlakACT line is indicated for integration into a normal hygiene routine, by preventing bacterial deposits (plaque) on the surface of the teeth and mucous membranes, keeping them in good condition. With three types of application (gel, spray and mouthwash) it acts exactly where you want it and with the intensity you want. PlakACT has been designed for use at home and in the dental practice for targeted administration by the practitioner.

For a free sample or more information on PlakACT Chlorhexidine line or to speak with a Kerr sales representative, visit https://www.kerrdental.com/en-uk/.

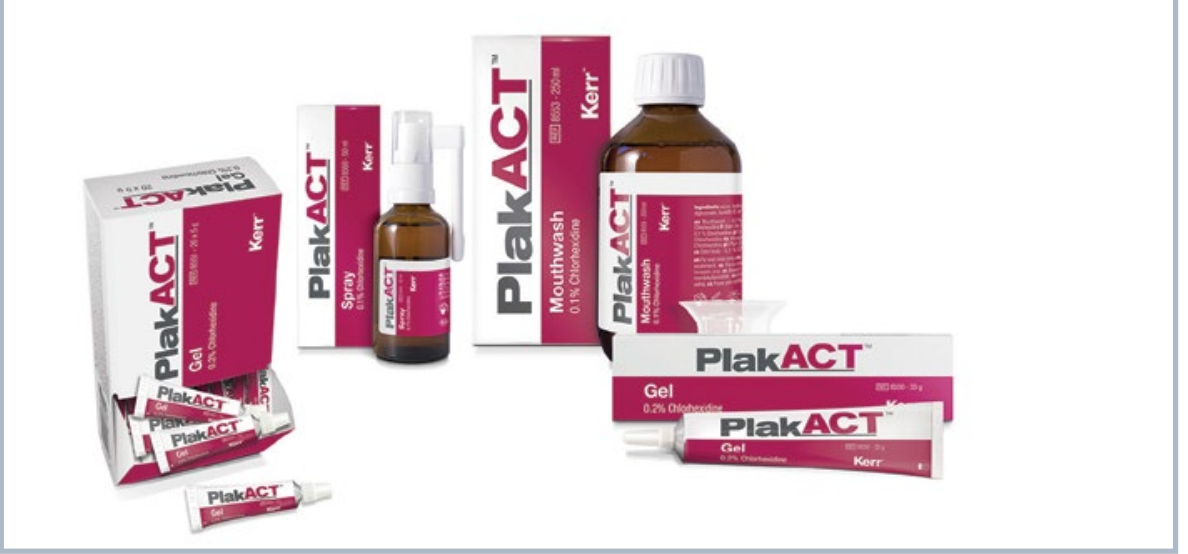

\section{New website for cloud dental patient software}

Take a tour of the new website https:// dentally.co/ designed to showcase why Dentally is the UK's leading cloud dental patient management software.

Dentally's website will allow you to navigate through some of our key features and find out how they deliver the best dental software on the market with cutting edge, cloud enabled tools.

Just like their software, Dentally have invested time in developing the new website to ensure it is completely customer centric, packed full of valuable information. Simply select your region and practice type to view content specifically related to the type of practice you run. Dentally will explain why they can provide you with an easier way of managing the many arms of dental practice life.

The website takes you through all of the key features from clinical, practice management, reporting, patient communications, reception tools and much more, so you can discover whether Dentally is a good match for your practice.

Visit the new website today to learn more https://dentally.co/ or email hello@dentally.co.

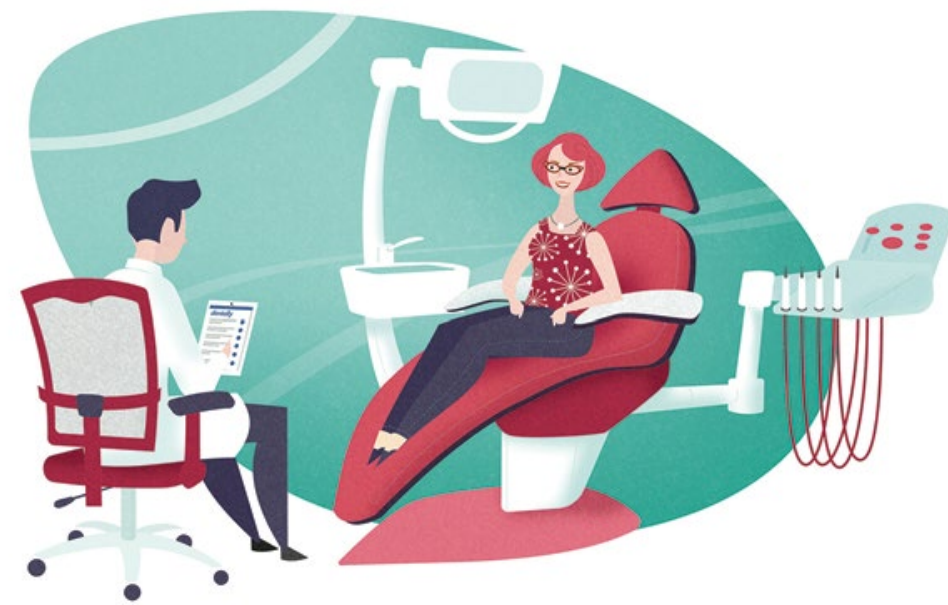

BRITISH DENTAL JOURNAL | VOLUME 229 NO. 8 | OCTOBER 232020 